\title{
Generalized Galitskii approach for the vertex function of a Fermi gas with resonant interaction
}

\author{
A. Vagov and H. Schomerus \\ Department of Physics, Lancaster University, Bailrigg, Lancaster LA1 4YB, United Kingdom
}

\section{A. Shanenko}

Departement Fysica, Universiteit Antwerpen, Groenenborgerlaan 171, B-2020 Antwerpen, Belgium

(Received 1 August 2007; revised manuscript received 22 October 2007; published 28 December 2007)

\begin{abstract}
We present a generalized Galitskii approach for the Bethe-Salpeter equation for the two-particle vertex function of a Fermi system with the resonant interaction by accounting for the resonant state in the scattering potential and utilizing the universal form of the resonant scattering amplitude. The procedure can be carried out both for the normal as well as for the condensate state. In both cases, the vertex function in the vicinity of the resonance is shown to formally coincide with that obtained for a weakly attractive Fermi gas. Thus we justify the popular calculational framework in which results for the weakly attractive Fermi gas are formally extrapolated into the domain of strong coupling, and further to the repulsive side of the resonance, where molecular states are formed.
\end{abstract}

DOI: 10.1103/PhysRevB.76.214513

PACS number(s): 03.75.Ss, 03.75.Hh

\section{INTRODUCTION}

The problem of an interacting Fermi system with strong resonant coupling is relevant to many areas of physics, in particular, ultracold gases ${ }^{1-3}$ and high $T_{c}$ superconductors. ${ }^{1}$ Experimental insight into this problem has recently been obtained using ultracold atomic ${ }^{6} \mathrm{Li}$ and ${ }^{40} \mathrm{~K}$ gases, ${ }^{2,3}$ in which the $s$-wave scattering length $a$ can be tuned from positive (repulsive) to negative (attractive) values by crossing a Feshbach resonance, at which the scattering length diverges. These experiments started with the successful formation of long-living paired fermions in the strong-coupling regime, ${ }^{4}$ which were subsequently seen to condense into a BoseEinstein condensate (BEC) of bound molecular states. ${ }^{5-7}$ Soon afterward the interaction was tuned to the attractive side ${ }^{8-11}$ and it was established that the condensate crosses over to a BCS superfluid of extended Cooper pairs. ${ }^{12-17}$

The first theoretical studies of the BCS-BEC crossover were initiated not long after the development of the BCS theory of superconductivity itself, when it was noticed that the nonzero solution of the BCS gap equation, complemented with the condition of a constant number of particles and regularized to eliminate an ultraviolet divergency, can be smoothly extrapolated to the domain of positive scattering lengths (without diverging at the resonance), where it describes molecular states. ${ }^{18-21}$

This mean-field interpolation between the two types of condensates is believed to be most accurate at $T=0$, where bosonic excitations of paired fermions (Cooper pairs or molecular states of finite momentum) are not populated. Subsequent works established that the pair excitations induce fluctuation corrections which modify the thermodynamics especially in the strong-coupling limit. ${ }^{22-27}$ In many of these works the fluctuation corrections are treated within the selfconsistent $T$-matrix approximation which involves the ladder approximation for the vertex function.

The calculations beyond the mean-field approximation in the BCS theory were initiated by Gorkov and MelikBarkhudarov; ${ }^{28}$ similar types of corrections were also ap- plied to the BCS-BEC crossover. ${ }^{29}$ Other recent approaches combine the ladder approximation with the fully selfconsistent solution for the single-particle Green's function, ${ }^{30}$ and account for the scattering of the weakly bound composite bosons. It should be noted that the scattering length for the two molecules in vacuo can be calculated exactly, ${ }^{31}$ providing a useful guidance for the many-body calculations. Furthermore, the BEC-BCS crossover can also be studied numerically in Monte Carlo simulations, ${ }^{32-34}$ or via the Renormalization Group analysis with $\varepsilon$ and $1 / N$ expansions at the unitary point. ${ }^{35-39}$

Comparison with experiment and Monte Carlo calculations reveals that the ladder approximation captures the qualitative features for various physical quantities in the BCS-BEC crossover far better than it could be expected. ${ }^{40,41}$ This is also astonishing since the corresponding BetheSalpeter equation is conventionally derived by assuming the constant approximation for the scattering matrix, ${ }^{42}$ which does not explicitly account for the resonant state. This approximation is strictly valid only for a weakly attracting Fermi gas. In order to study the strong-coupling limit, the resulting expressions are formally extrapolated, and these expressions are also used on the repulsive side of the resonance. Curiously, they then capture molecular states of the correct energy, and also recover the scattering length for the molecules in the Born approximation. Moreover, the results turn out to be nondivergent at resonance. These observations have motivated several works which address the justification of solving the Bethe-Salpeter equation for the strongcoupling case and in the presence of bound molecular states. In the context of high- $T_{c}$ two-dimensional superconductors the validity of the extrapolation procedure for the strongcoupling regime was discussed by Randeria et al.,${ }^{43}$ while for the ultracold Fermi gases the properties of the resonant scattering amplitude were used by Combescot ${ }^{44}$ to search for the universal form of the scattering matrix which would be applicable close to the resonance while recovering both limiting cases far from it.

In this work we solve the Bethe-Salpeter equation for the two-particle vertex function of a Fermi system near the reso- 
nance, and show that the resulting expression coincides with the result of the extrapolation and regularization procedure. Our derivation proceeds via an extension of the Galitskii formalism, ${ }^{42}$ which reformulates the Bethe-Salpeter equation by using the scattering amplitude instead of the scattering potential. In its original version, the Galitskii formalism is unsuitable to describe resonant scattering, since it does not explicitly account for the resonant state and as a result treats the case of positive scattering length $a$ as a repulsive Fermi system. In our derivation we do not use any assumptions beyond the condition that the resonant state induces a broad Feshbach resonance. The state is eliminated by using the completeness and orthogonality of the set of all scattering states. This procedure can be carried out both for the normal state as well as for the condensate state, and leads to a fully analytical solution of the Bethe-Salpeter equation which is inherently valid in the vicinity of the resonance, as well as in the domain of the positive scattering length. The resulting expressions of this formal derivation turn out to be identical to the BCS extrapolation scheme with the renormalized contact interaction. Furthermore, our derivation constitutes a reorganization of the ladder approximation which demonstrates that the relevant expansion parameter does not diverge at resonance. Thus we establish a firm basis for the common conceptual framework which is used both in the mean-field description of the BCS-BEC crossover, as well as in many calculations accounting for the fluctuation corrections.

This paper is organized as follows. Section II defines the microscopic Fermi model on which all considerations are based. In Sec. III we present the modified Galitskii formalism for the Fermi gas in the normal state. In Sec. IV we present the derivation for the Fermi system in the condensate state, and also briefly discuss the gap equation. The results of this paper are summarized in Sec. V.

\section{SINGLE-CHANNEL MODEL FOR THE RESONANTLY INTERACTING FERMI GAS}

In order to describe an ultracold gas of fermionic atoms in the vicinity of a Feshbach broad resonance we use the standard single-channel Hamiltonian

$$
\begin{aligned}
H= & \sum_{\alpha} \int d x\left[\psi_{\alpha}^{\dagger}(\mathbf{r})\left(-\frac{\hbar^{2} \nabla^{2}}{2 m}-\mu\right) \psi_{\alpha}(\mathbf{r})\right] \\
& +\frac{1}{2} \sum_{\alpha, \beta} \int d \mathbf{r} d \mathbf{r}^{\prime} \psi_{\alpha}^{\dagger}(\mathbf{r}) \psi_{\beta}^{\dagger}\left(\mathbf{r}^{\prime}\right) U_{\alpha \beta}\left(\mathbf{r}-\mathbf{r}^{\prime}\right) \psi_{\beta}\left(\mathbf{r}^{\prime}\right) \psi_{\alpha}(\mathbf{r}),
\end{aligned}
$$

where $\psi_{\alpha}(\mathbf{r})$ is a fermionic field operator for a particle with spin $\alpha$. In the following we work in the units $m \equiv 1, \hbar \equiv 1$, and also set Boltzmann's constant $k_{B} \equiv 1$. The effective scattering potential $U_{\alpha \beta}(\mathbf{r})$ describes resonant scattering of particles of opposite spin in the $s$-wave channel. In the vicinity of a broad resonance with effective interaction radius $r_{0} \ll k_{F}^{-1}$ (where $k_{F}$ is the Fermi momentum), the scattering amplitude in the $s$-wave channel takes the universal form ${ }^{45}$

$$
f(\mathbf{k}) \approx-\frac{1}{\eta+i|\mathbf{k}|},
$$

where $\eta=a^{-1}$ is the inverse scattering length. The scattering length $a$ diverges at resonance, $\eta=0$. For $\eta<0$ the resonant level lies in the continuum, and the interaction is attractive. For $\eta>0$ the resonant level turns into a bound state, and the interaction is repulsive. In the BCS limit $\eta \rightarrow-\infty$, the interaction mediates the formation of Cooper pairs, which are weakly bound in momentum space. In the BEC limit $\eta \rightarrow+\infty$, the interaction mediates the formation of molecular states, which are weakly bound in real space. Because these notions refer to the condensate phase, we more broadly speak of the Fermi limit for $\eta \rightarrow-\infty$, and of the Bose limit for $\eta \rightarrow+\infty$.

It should be stressed that the Hamiltonian (1) is purely fermionic, and can be contrasted to the more detailed FermiBose models which explicitly account for a bound molecular state in the closed channel. ${ }^{25,46-48}$ A great deal of work has been carried out in the past to give a detailed justification of the validity of both models and, in particular, to show their equivalence in the strong-coupling regime of a broad Feshbach resonance. ${ }^{49-54}$ The universality arises for a combination of two facts. First, at $\eta=0$ the scattering length diverges, which renders the Fermi energy $E_{F}=k_{F}^{2} / 2$ as the only relevant energy scale for the thermodynamics. ${ }^{55}$ Second, in this unitary limit the contribution of the closed channel becomes negligible, which has been directly tested in experiments on ${ }^{6} \mathrm{Li},{ }^{56}$ even though the situation in ${ }^{40} \mathrm{~K}$ may be a matter of debate. ${ }^{57,58}$

\section{MODIFIED GALITSKII FORMALISM FOR RESONANT SCATTERING}

We first assume that the Fermi gas is in the normal state. In the ladder approximation, the vertex function $\Gamma\left(p_{1}, p_{2} ; p_{3}, p_{4}\right)$ is then determined by the Bethe-Salpeter equation

$$
\begin{aligned}
\Gamma\left(p_{1}, p_{2} ; p_{3}, p_{4}\right)= & u\left(\mathbf{p}_{1}-\mathbf{p}_{3}\right)-T \sum_{q_{0}} \int \frac{d \mathbf{q}}{(2 \pi)^{3}} u(\mathbf{q}) G\left(p_{1}-q\right) \\
& \times G\left(p_{2}+q\right) \Gamma\left(p_{1}-q, p_{2}+q ; p_{3}, p_{4}\right),
\end{aligned}
$$

where spin indices are omitted for clarity, $p_{i}=\left(p_{i, 0}, \mathbf{p}\right)$ is the four-dimensional energy-momentum vector of an incoming $(i=1,2)$ or outgoing $(i=3,4)$ particle, $u(\mathbf{q})$ is the momentum representation of the interaction potential, and

$$
G(q)=\frac{1}{q_{0}-\xi_{\mathbf{q}}}, \quad \xi_{\mathbf{q}}=\frac{\mathbf{q}^{2}}{2}-\mu
$$

is the Green's function of a noninteracting Fermi system. We use the notation $q=\left(q_{0}, \mathbf{q}\right)$, where $\mathbf{q}$ denotes the threedimensional momentum vector and $q_{0}=i \pi T(2 n+1)$ is the Matsubara frequency.

In solving Eq. (3) one follows the conventional strategy, ${ }^{42}$ developed for the $T=0$ case, where the potential $u(\mathbf{q})$ is eliminated in favor of the vacuo vertex function $\Gamma_{0}$, defined as the solution of Eq. (3) for two particles in absence of all 
the other particles, i.e., by setting $\mu=0$. An extension of this strategy for the finite temperature case is conveniently achieved by a subsequent analytic continuation of the energy argument in $\Gamma_{0}$ as

$$
E=g_{0}+2 \mu-\mathbf{g}^{2} / 4 .
$$

Hereafter, we denote the total momentum of the scattered particles as $g=p_{1}+p_{2}=\left(g_{0}, \mathbf{g}\right)$, while $p=\left(p_{1}-p_{2}\right) / 2$ and $p^{\prime}=\left(p_{3}-p_{4}\right) / 2$ are the relative momenta before and after the scattering event, respectively.

Eliminating $u(\mathbf{p})$ in Eq. (3), the Bethe-Salpeter equation takes the form

$$
\begin{aligned}
\Gamma\left(\mathbf{p}, \mathbf{p}^{\prime}, g\right)= & \Gamma_{0}\left(\mathbf{p}, \mathbf{p}^{\prime}, g\right)-\int \frac{d^{3} \mathbf{q}}{(2 \pi)^{3}} \Gamma_{0}(\mathbf{p}, \mathbf{q}, g) \\
& \times K(\mathbf{q}, g) \Gamma\left(\mathbf{q}, \mathbf{p}^{\prime}, g\right) .
\end{aligned}
$$

The kernel $K$ is defined as

$$
K(\mathbf{q}, g)=T \sum_{q_{0}} G\left(\frac{\mathbf{g}}{2}-\mathbf{q}, g_{0}-q_{0}\right) G\left(\frac{\mathbf{g}}{2}+q, q_{0}\right)+\frac{1}{E-\mathbf{q}^{2}} .
$$

Using Eq. (4) one obtains an explicit expression of the kernel,

$$
K(\mathbf{q}, g)=\frac{n_{F}\left(\xi_{+}\right)+n_{F}\left(\xi_{-}\right)}{E-\mathbf{q}^{2}},
$$

where $n_{F}(\varepsilon)=[\exp (\varepsilon / T)+1]^{-1}$ is the Fermi function and

$$
\xi_{ \pm}=\frac{1}{2}\left(\frac{\mathbf{g}}{2} \pm \mathbf{q}\right)^{2}-\mu
$$

In the Galitskii approach (see, e.g., Ref. 42), the vacuo vertex function $\Gamma_{0}$ is found by relating it to the twomomentum scattering amplitude $f(\mathbf{p}, \mathbf{k})$. However, the original formalism does not account for a possible bound molecular state in the scattering potential. In the following we assume that the scattering potential $u(\mathbf{q})$ allows for a single bound state, with eigenfunction $\phi(x)$ and eigenvalue $\lambda$.

In the first step, $\Gamma_{0}$ is written as an integral

$$
\Gamma_{0}\left(\mathbf{p}, \mathbf{p}^{\prime}, g\right)=\int \frac{d^{3} \mathbf{q}}{(2 \pi)^{3}} u(\mathbf{q}) \chi\left(\mathbf{p}-\mathbf{q}, \mathbf{p}^{\prime}, g\right),
$$

where the scattering state $\chi\left(\mathbf{p}, \mathbf{p}^{\prime}, g\right)$ satisfies the equation

$$
\begin{aligned}
(E- & \left.\mathbf{p}^{2}+i 0\right) \chi\left(\mathbf{p}, \mathbf{p}^{\prime}, g\right)-\int \frac{d^{3} \mathbf{q}}{(2 \pi)^{3}} u(\mathbf{q}) \chi\left(\mathbf{p}-\mathbf{q}, \mathbf{p}^{\prime}, g\right) \\
& =(2 \pi)^{3}\left(E-\mathbf{p}^{2}+i 0\right) \delta\left(\mathbf{p}-\mathbf{p}^{\prime}\right) .
\end{aligned}
$$

The left-hand side of this equation is equivalent to a Schrödinger equation in momentum space. Therefore, $\chi\left(\mathbf{p}, \mathbf{p}^{\prime}, g\right)$ can be written using the complete set of solutions of the Schrödinger equation,

$$
\begin{aligned}
\chi\left(\mathbf{p}, \mathbf{p}^{\prime}, g\right)= & \left(E-\mathbf{p}^{\prime 2}+i 0\right) \int \frac{d^{3} \mathbf{k}}{(2 \pi)^{3}} \frac{\psi_{\mathbf{k}}(\mathbf{p}) \psi_{\mathbf{k}}^{*}\left(\mathbf{p}^{\prime}\right)}{E-\mathbf{k}^{2}+i 0} \\
& +\frac{E-\mathbf{p}^{\prime 2}+i 0}{E-2 \lambda+i 0} \phi(\mathbf{p}) \phi^{*}\left(\mathbf{p}^{\prime}\right),
\end{aligned}
$$

where $\psi_{\mathbf{k}}(\mathbf{p})$ is the Fourier transform of the scattering state $\psi_{\mathbf{k}}(\mathbf{r})$ with momentum $\mathbf{k}$. The last term of this expression explicitly accounts for the bound state $\phi(\mathbf{p})$. The states $\psi_{\mathbf{k}}(\mathbf{p})$ can be expressed via the two-momentum scattering amplitude $f(\mathbf{p}, \mathbf{k})$,

$$
\psi_{\mathbf{k}}(\mathbf{p})=(2 \pi)^{3} \delta(\mathbf{p}-\mathbf{k})+\frac{f(\mathbf{p}, \mathbf{k})}{\mathbf{k}^{2}-\mathbf{p}^{2}+i 0} .
$$

Inserting this expression into Eq. (12) one obtains

$$
\begin{aligned}
\chi\left(\mathbf{p}, \mathbf{p}^{\prime}, g\right)= & \psi_{\mathbf{p}^{\prime}}(\mathbf{p})+\int \frac{d^{3} \mathbf{k}}{(2 \pi)^{3}} \psi_{\mathbf{k}}(\mathbf{p}) f^{*}\left(\mathbf{p}^{\prime}, \mathbf{k}\right)\left(\frac{1}{E-\mathbf{k}^{2}+i 0}\right. \\
& \left.+\frac{1}{\mathbf{k}^{2}-\mathbf{p}^{\prime 2}-i 0}\right)+\frac{E-\mathbf{p}^{\prime 2}+i 0}{E-2 \lambda+i 0} \phi(\mathbf{p}) \phi^{*}\left(\mathbf{p}^{\prime}\right) .
\end{aligned}
$$

After substituting Eq. (14) into Eq. (10) one obtains $\Gamma_{0}$ as a sum of two terms

$$
\Gamma_{0}\left(\mathbf{p}, \mathbf{p}^{\prime}, g\right)=\Gamma_{0}^{G}\left(\mathbf{p}, \mathbf{p}^{\prime}, g\right)+\Gamma_{0}^{b}\left(\mathbf{p}, \mathbf{p}^{\prime}, g\right) .
$$

The first term in Eq. (15) recovers the original Galitskii result

$$
\begin{aligned}
\Gamma_{0}^{G}\left(\mathbf{p}, \mathbf{p}^{\prime}, g\right)= & f\left(\mathbf{p}, \mathbf{p}^{\prime}\right)+\int \frac{d^{3} \mathbf{k}}{(2 \pi)^{3}} f(\mathbf{p}, \mathbf{k}) f^{*}\left(\mathbf{p}^{\prime}, \mathbf{k}\right) \\
& \times\left(\frac{1}{E-\mathbf{k}^{2}+i 0}+\frac{1}{\mathbf{k}^{2}-\mathbf{p}^{\prime 2}-i 0}\right) .
\end{aligned}
$$

The second term in Eq. (15) originates from the bound state and is given by

$$
\Gamma_{0}^{b}\left(\mathbf{p}, \mathbf{p}^{\prime}, g\right)=\frac{E-\mathbf{p}^{\prime 2}}{E-2 \lambda} \int \frac{d^{3} \mathbf{q}}{(2 \pi)^{3}} u(\mathbf{q}) \phi(\mathbf{p}-\mathbf{q}) \phi^{*}\left(\mathbf{p}^{\prime}\right) .
$$

This expression still explicitly depends on the bound state, and at first glance it appears that detailed knowledge of $\phi(\mathbf{p})$ is necessary in subsequent calculations. However, $\phi(\mathbf{p})$ can be eliminated by relating it to $f(\mathbf{k}, \mathbf{p})$. To establish this relation we utilize the Schrödinger equation for $\phi(\mathbf{p})$ in the momentum representation

$$
\left(2 \lambda-\mathbf{p}^{2}\right) \phi(\mathbf{p})=\int \frac{d^{3} \mathbf{q}}{(2 \pi)^{3}} u(\mathbf{q}) \phi(\mathbf{p}-\mathbf{q})
$$

and the completeness relation

$$
\int \frac{d^{3} \mathbf{k}}{(2 \pi)^{3}} \psi_{\mathbf{k}}(\mathbf{p}) \psi_{\mathbf{k}}^{*}\left(\mathbf{p}^{\prime}\right)+\phi(\mathbf{p}) \phi^{*}\left(\mathbf{p}^{\prime}\right)=(2 \pi)^{3} \delta\left(\mathbf{p}-\mathbf{p}^{\prime}\right) .
$$

Multiplying both sides of Eq. (17) by $\phi(\mathbf{p})$, then using Eq. (19) and the definition (13) one obtains the correction of the 
Galitskii result due to the contribution of the molecular state as

$$
\begin{aligned}
\Gamma_{0}^{b}\left(\mathbf{p}, \mathbf{p}^{\prime}, g\right)= & \frac{E-\mathbf{p}^{\prime 2}}{E-2 \lambda} \frac{2 \lambda-\mathbf{p}^{2}}{\mathbf{p}^{2}-\mathbf{p}^{\prime 2}-i 0}\left[\int \frac{d^{3} \mathbf{k}}{(2 \pi)^{3}} f(\mathbf{p}, \mathbf{k}) f^{*}\left(\mathbf{p}^{\prime}, \mathbf{k}\right)\right. \\
& \times\left(\frac{1}{\mathbf{k}^{2}-\mathbf{p}^{\prime 2}-i 0}-\frac{1}{\mathbf{k}^{2}-\mathbf{p}^{2}+i 0}\right) \\
& \left.+f\left(\mathbf{p}, \mathbf{p}^{\prime}\right)-f^{*}\left(\mathbf{p}^{\prime}, \mathbf{p}\right)\right] .
\end{aligned}
$$

Equations (15), (16), and (20) reduce the calculation of the vacuo vertex to that for the two-momentum scattering amplitude $f(\mathbf{p}, \mathbf{k})$, defined by Eq. (13). This amplitude is obtained by solving the Lippmann-Schwinger equation. In the weak-scattering limit, $f(\mathbf{p}, \mathbf{k})$ can be approximated by a constant $4 \pi / \eta$. Following Eq. (15), this leads to a constant approximation for $\Gamma_{0}$ as well. Since the vertex function then does not possess any poles, the constant approximation cannot be used to describe the bound states. In a more accurate analysis, applicable close to the resonance as well as in the limits $\eta \rightarrow \pm \infty$, one can utilize the fact that in the vicinity of a resonance the two-particle scattering amplitude for $s$-wave scattering $f(\mathbf{p}, \mathbf{k})$ assumes as similar universal form as the single-particle scattering amplitude $f(\mathbf{k}),{ }^{45}$

$$
f(\mathbf{p}, \mathbf{k})=\frac{4 \pi}{\eta+i|\mathbf{k}|}
$$

where in general $\eta$ depends on $\mathbf{p}$ and $\mathbf{k}$. For small momenta, $\eta$ approaches a constant equal to the inverse scattering length. The leading corrections are quadratic, $\propto \mathbf{k}^{2}$ and $\propto \mathbf{p}^{2}$, but the coefficients for these terms are proportional to the effective radius of the scattering potential $r_{0} .{ }^{42,45}$ Under the condition of a broad resonance one has $r_{0} k_{F} \ll 1$. Therefore, these terms can be neglected and $\eta$ in Eq. (21) can be assumed constant, equal to the inverse scattering length. On the molecular side of the resonance, where the potential permits a bound state, the inverse scattering length is furthermore related to the bound state energy via $2 \lambda=-\eta^{2} .{ }^{45}$

It should be noted that Eq. (21) reduces to the correct constant expressions in the weak-coupling limits $\eta \rightarrow \pm \infty$, and also satisfies the optical theorem; as we will see later, these observations are strongly linked to the success of the conventional extrapolation-and-regularization procedure for the vertex function.

Substituting Eq. (21) into Eq. (16) when $\eta<0$ and into Eqs. (16) and (20) when $\eta>0$ and using $2 \lambda=-\eta^{2}$ one obtains a simple expression for the vacuo vertex function as

$$
\Gamma_{0}(E)=\frac{4 \pi}{\eta+i \sqrt{E}},
$$

where the branch cut of the square root lies on the real semi axis $E>0$, such that on the first Riemann sheet $i \sqrt{-1}=-1$. Recalling the definition of $E$ one sees that the vacuo vertex function only depends on the total momentum and energy of the molecular pair. Equation (22) is applicable on both sides of the resonance and does not have any singularity at the resonance. On the repulsive side $\eta>0$, Eq. (22) has a pole at
$E=-\eta^{2}$, which describes a bound state with binding energy $\eta^{2}$. On the attractive side $\eta<0$, the pole is absent: it is located on the unphysical second Riemann sheet of complex energy $E$. At resonance, Eq. (22) yields a square root singularity, which is different from the simple pole obtained in Ref. 44.

For comparison it is instructive to recalculate $\Gamma_{0}$ for a repulsive potential $\eta>0$ using the original Galitskii result (16), i.e., without the bound state. In this case one obtains a different expression

$$
\Gamma_{0}\left(\mathbf{p}^{\prime}, E\right)=4 \pi\left(\frac{2 \eta}{\eta^{2}+\mathbf{p}^{\prime 2}}-\frac{1}{\eta-i \sqrt{E}}\right) .
$$

Contrary to Eq. (22), Eq. (23) does not have a pole that corresponds to the bound state. Equations (22) and (23) explicitly distinguish between potentials that have the same scattering length but do or do not permit a bound state, respectively. We note that the series expansion of Eqs. (22) and (23) with respect to large $\eta$ differs only in the third-order term. Therefore, both Eqs. (22) and (23) yield the same result when the Bethe-Salpeter equation (6) is solved in a secondorder approximation, as done in the original Galitskii formalism. ${ }^{42}$

We now proceed with the solution of Eq. (6) for the twoparticle scattering amplitude (22). In doing so, we perform an analytical continuation as defined by Eq. (5). The solution can then be written as

$$
\Gamma(g)=\left[\Gamma_{0}^{-1}+K(g)\right]^{-1}=\frac{4 \pi}{\eta+i \sqrt{E}+4 \pi K(g)},
$$

where

$$
K(g)=\int \frac{d^{3} \mathbf{q}}{(2 \pi)^{3}} \frac{\tanh \left(\xi_{+} / 2 T\right)+\tanh \left(\xi_{-} / 2 T\right)-2}{2\left(\mathbf{q}^{2}-E\right)} .
$$

In the limit $\eta \rightarrow \infty, \mu \rightarrow-\infty$, where the system is dominated by the molecular states, the vertex (24) reduces to the vacuo vertex function (22).

In the final step of the derivation we integrate the last term in Eq. (25) by parts. This cancels the term $i \sqrt{E}$ in Eq. (24), yielding

$$
\Gamma(g)=\frac{4 \pi}{\eta+\tilde{K}(g)},
$$

where

$$
\tilde{K}(g)=\int \frac{d^{3} \mathbf{q}}{4 \pi^{2}}\left[\frac{\tanh \left(\xi_{+} / 2 T\right)+\tanh \left(\xi_{-} / 2 T\right)}{\mathbf{q}^{2}-E}-\frac{2}{\mathbf{q}^{2}}\right] .
$$

This expression coincides precisely with the widely used extrapolated weak-coupling result, ${ }^{1,2}$ including the ultraviolet regularization for the contact interaction (see, e.g., Ref. 59). The derivation procedure above demonstrated that in the ladder approximation, this expression remains strictly valid in the vicinity of the resonance, as well as on the molecular side $\eta>0$. The reason for the fact that the vertex function (26a) and (26b), originally derived in the limit $\eta \rightarrow-\infty$, correctly 
describes a molecular bound state for $\eta>0$ lies in the fact that $\Gamma$ approaches $\Gamma_{0}$ in Eq. (22). There is also another, more intuitive reason why the formal extrapolation of the results for the weakly interacting Fermi limit to the domain $\eta>0$ correctly describes the case with a molecular bound state and not the weakly repulsive Fermi gas. For a purely repulsive potential without such a state, the resonant limit $\eta \rightarrow 0$ can only be realized when the effective radius of the potential also becomes infinite. This would be in conflict with the continuity of the vertex function (26a) and (26b) as a function of $\eta$, which also holds across the resonance at $\eta=0$.

\section{VERTEX FUNCTION IN THE CONDENSATE STATE}

The modified Galitskii approach described above can be equally applied to solve the Bethe-Salpeter equation for the vertex function in the condensate system. The condensate system has normal as well as anomalous vertex functions (with four incoming or outgoing lines) and the resulting Bethe-Salpeter equation becomes a system of equations ${ }^{60}$

$$
\begin{aligned}
\Gamma\left(p_{1}, p_{2} ; p_{3}, p_{4}\right) \\
=u\left(\mathbf{p}_{1}-\mathbf{p}_{2}\right)-T \sum_{q_{0}} \int \frac{d^{3} \mathbf{q}}{(2 \pi)^{3}} u(\mathbf{q})\left[G\left(p_{1}-q\right) G\left(p_{2}+q\right)\right. \\
\quad \times \Gamma\left(p_{1}-q, p_{2}+q ; p_{3}, p_{4}\right)+F\left(p_{1}-q\right) F\left(p_{2}+q\right) \\
\left.\quad \times \Theta\left(p_{1}-q, p_{2}+q ; p_{3}, p_{4}\right)\right], \\
\Theta\left(p_{1}, p_{2} ; p_{3}, p_{4}\right) \\
=-T \sum_{q_{0}} \int \frac{d^{3} \mathbf{q}}{(2 \pi)^{3}} u(\mathbf{q})\left[G\left(q-p_{1}\right) G\left(-p_{2}-q\right)\right. \\
\quad \times \Theta\left(p_{1}-q, p_{2}+q ; p_{3}, p_{4}\right)+F^{*}\left(p_{1}-q\right) F^{*}\left(p_{2}-q\right) \\
\left.\quad \times \Gamma\left(p_{1}-q, p_{2}+q ; p_{3}, p_{4}\right)\right] .
\end{aligned}
$$

The normal and anomalous Green's functions $G$ and $F$, respectively, are defined as

$$
G(q)=\frac{q_{0}+\xi_{\mathbf{q}}}{q_{0}^{2}-\Delta_{\mathbf{q}}^{2}}, \quad F(q)=\frac{\Delta}{q_{0}^{2}-\Delta_{\mathbf{q}}^{2}},
$$

where $\Delta$ is the BCS single-particle spectral gap, related to the condensate density, and

$$
\Delta_{\mathbf{q}}=\sqrt{\xi_{\mathbf{q}}^{2}+\Delta^{2}},
$$

while $\xi_{\mathbf{q}}$ is given in Eq. (4). Solution of this system along the lines of the Galitskii formalism outlined in the previous section yields the algebraic system

$$
\begin{gathered}
\Gamma(g)=\Gamma_{0}(g)[1-K(g) \Gamma(g)-S(g) \Theta(g)], \\
\Theta(g)=-\Gamma_{0}(-g)\left[K(-g) \Theta(g)+S^{*}(g) \Gamma(g)\right],
\end{gathered}
$$

where the kernel $K(\mathrm{~g})$ is defined as the integral of Eq. (7) over $\mathbf{k}$ and the Green's function, $G$, defined by Eq. (30) while $S(g)$ is

$$
S(g)=\int \frac{d^{3} \mathbf{q}}{(2 \pi)^{3}} T \sum_{k_{0}} F\left(\frac{g}{2}-q\right) F\left(\frac{g}{2}+q\right) .
$$

The algebraic pair of Eq. (32) is solved by

$$
\Gamma(g)=\frac{\eta+i \sqrt{E}+4 \pi K(-g)}{\Xi(g)}, \quad \Theta(g)=\frac{4 \pi S^{*}(g)}{\Xi(g)},
$$

where the common denominator is given by

$$
\begin{aligned}
\Xi(g)= & {[\eta+i \sqrt{E}+4 \pi K(g)][\eta+i \sqrt{E}+4 \pi K(-g)] } \\
& -16 \pi^{2} S(g) S(g)^{*} .
\end{aligned}
$$

As in the normal case the partial integration in the normal kernel removes $i \sqrt{E}$ and the result for the vertex becomes equivalent to that in the weakly coupled neutral BCS system, which again by the virtue of the derivation is valid for arbitrary $\eta$, including $\eta=0$.

We conclude the analysis by a brief discussion of the gap equation, which determines the order parameter $\Delta$. According to the Thouless criterion, the stability of the condensate requires that the bosonic excitation spectrum defined by the poles of $\Gamma$ is gapless. This yields

$$
\eta+i \sqrt{E}+4 \pi K(0)+4 \pi S(0)=0 .
$$

[The second possible equation with the minus sign in front of $S(0)$ yields instabilities in the corresponding bosonic excitation spectrum.] The explicit form of Eq. (35) is

$$
-\eta=\frac{2}{\pi} \int_{0}^{\infty} d q\left[\frac{q^{2}}{2 \Delta_{q}} \tanh \left(\frac{\Delta_{q}}{2 T}\right)-1\right] .
$$

This is the familiar BCS gap equation for a weakly interacting BCS system. However, as the underlying vertex function (33), Eq. (36) is valid (within the mean-field approximation) in the vicinity of the resonance as well as on its repulsive side, $\eta>0$. The observation that at $\eta>0$ this mean-field gap equation, combined with the condition of the constant particle density, correctly describes the bound molecular states (see, e.g., Refs. 18 and 21) is again ensured by the correct limit $\Gamma \rightarrow \Gamma_{0}$ of the vertex function in the molecular limit $\eta \rightarrow \infty, \mu \rightarrow-\infty$.

\section{CONCLUSIONS}

In this work we have presented a procedure of solving the Bethe-Salpeter equation for the two-particle vertex functions in the ladder approximation in the vicinity of a broad resonance, as encountered for ultracold Fermi gases close to a Feshbach resonance. In order to do this we have extended the Galitskii formalism to account for the molecular states in the interaction potential and also utilized the universal form of the resonant scattering amplitude in the vicinity of the resonance. This allowed for the exact solution of the BetheSalpeter equation at resonance and its vicinity. It is also valid in the weak-scattering limit, including the limit of the molecular states. The solution is regular in the BCS-BEC crossover and coincides exactly with the standard results obtained for the weakly attractive Fermi gas. This agreement provides 
a link between the resonance regime and the weakinteraction regime and serves to justify the widely used calculational framework in which the weak-interaction results (with ultraviolet regularization) are extrapolated to the BCS$\mathrm{BEC}$ crossover and further to the domain of molecular states.

The derivation of the vertex function can be carried out both in the normal as well as in the condensate state. As the latter is used to extract the equation for the condensate density (the gap equation), the extrapolation procedure for the BCS gap equation onto the molecular side of the resonance is also perfectly justified (within the ladder approximation). Thus our results establish the robustness of the phenomenological extrapolation from the BCS limit used in many previous works.

We have not discussed the validity of the ladder approximation itself, which is obviously questionable when the system is close to the resonance. The applicability of the ladder approximation is conventionally established for a small gas parameter $k_{F} /|\eta| \ll 1$. This condition apparently fails at resonance, where the gas parameter diverges. Still, it has been found that the ladder approximation combined with a selfconsistency procedure delivers reliable qualitative, and to some extent even quantitative information in the complete crossover regime (see, e.g., Refs. 1 and 2). The presented solution of the Bethe-Salpeter equations in the vicinity of the resonance reveals that the relevant small parameter in the resonant system is $\propto k_{F}\left|f\left(k_{F}\right)\right|$, where $\left|f\left(k_{F}\right)\right|$ is the absolute value of the scattering amplitude. This quantity coincides with the conventional gas parameter in the weak-interaction limit, and, although not small, is not divergent at resonance, approaching a constant of order unity instead. While not providing a small expansion parameter, this observation provides further insight into the apparent successes of the ladder approximation in studies of the resonant scattering systems.

\section{ACKNOWLEDGMENT}

This work was supported by the European Commission, Marie Curie Excellence Grant MEXT-CT-2005-023778 (Nanoelectrophotonics).
${ }^{1}$ Q. Chen, J. Stajic, S. Tan, and K. Levin, Phys. Rep. 412, 1 (2005).

${ }^{2}$ For recent developments in the physics of ultracold gases see, e.g., I. Bloch, J. Dalibard, and W. Zwerger, arXiv:0704.3011, Rev. Mod. Phys. (to be published).

${ }^{3}$ W. Ketterle and Y. Shin, Phys. World 20 (6), 39 (2007).

${ }^{4}$ J. Cubizolles, T. Bourdel, S. J. J. M. F. Kokkelmans, G. V. Shlyapnikov, and C. Salomon, Phys. Rev. Lett. 91, 240401 (2003).

${ }^{5}$ M. Greiner, C. A. Regal, and D. S. Jin, Nature (London) 426, 537 (2003)

${ }^{6}$ M. W. Zwierlein, C. A. Stan, C. H. Schunck, S. M. F. Raupach, S. Gupta, Z. Hadzibabic, and W. Ketterle, Phys. Rev. Lett. 91, 250401 (2003).

${ }^{7}$ S. Jochim, M. Bartenstein, A. Altmeyer, G. Hendl, S. Riedl, C. Chin, J. Hecker Denschlag, and R. Grimm, Science 302, 2101 (2003).

${ }^{8}$ C. A. Regal, M. Greiner, and D. S. Jin, Phys. Rev. Lett. 92, 040403 (2004).

${ }^{9}$ M. Bartenstein, A. Altmeyer, S. Riedl, S. Jochim, C. Chin, J. H. Denschlag, and R. Grimm, Phys. Rev. Lett. 92, 120401 (2004).

${ }^{10}$ M. W. Zwierlein, C. A. Stan, C. H. Schunck, S. M. F. Raupach, A. J. Kerman, and W. Ketterle, Phys. Rev. Lett. 92, 120403 (2004).

${ }^{11}$ T. Bourdel, L. Khaykovich, J. Cubizolles, J. Zhang, F. Chevy, M. Teichmann, L. Tarruell, S. J. J. M. F. Kokkelmans, and C. Salomon, Phys. Rev. Lett. 93, 050401 (2004).

${ }^{12}$ J. Kinast, S. L. Hemmer, M. E. Gehm, A. Turlapov, and J. E. Thomas, Phys. Rev. Lett. 92, 150402 (2004).

${ }^{13}$ M. Bartenstein, A. Altmeyer, S. Riedl, S. Jochim, C. Chin, J. H. Denschlag, and R. Grimm, Phys. Rev. Lett. 92, 203201 (2004).

${ }^{14}$ C. Chin, M. Bartenstein, A. Altmeyer, S. Riedl, S. Jochim, J. H. Denschlag, and R. Grimm, Science 305, 1128 (2004).

${ }^{15}$ M. W. Zwierlein, J. R. Abo-Shaeer, A. Schirotzek, C. H. Schunck, and W. Ketterle, Nature (London) 435, 1047 (2005).

${ }^{16}$ M. W. Zwierlein, C. H. Schunck, A. Schirotzek, and W. Ketterle,
Nature (London) 442, 54 (2006).

${ }^{17}$ A. Altmeyer, S. Riedl, C. C. Kohstall, M. J. Wright, R. Geursen, M. Bartenstein, C. Chin, J. H. Denschlag, and R. Grimm, Phys. Rev. Lett. 98, 040401 (2007).

${ }^{18}$ D. M. Eagles, Phys. Rev. 186, 456 (1969).

${ }^{19}$ A. J. Leggett, in Modern Trends in the Theory of Condensed Matter, edited by A. Pekalski and R. Przystawa (Springer, Berlin, 1980).

${ }^{20}$ J. R. Engelbrecht, M. Randeria, and C. A. R. Sá de Melo, Phys. Rev. B 55, 15153 (1997).

${ }^{21}$ L. Viverit, S. Giorgini, L. P. Pitaevskii, and S. Stringari, Phys. Rev. A 69, 013607 (2004).

${ }^{22}$ P. Nozières and S. Schmitt-Rink, J. Low Temp. Phys. 59, 195 (1985).

${ }^{23}$ R. Haussmann, Z. Phys. B: Condens. Matter 91, 291 (1993).

${ }^{24}$ R. Haussmann, Phys. Rev. B 49, 12975 (1994).

${ }^{25}$ Y. Ohashi and A. Griffin, Phys. Rev. Lett. 89, 130402 (2002).

${ }^{26}$ P. Pieri, L. Pisani, and G. C. Strinati, Phys. Rev. B 70, 094508 (2004).

${ }^{27}$ R. Combescot, X. Leyronas, and M. Yu. Kagan, Phys. Rev. A 73, 023618 (2006).

${ }^{28}$ L. P. Gorkov and T. K. Melik-Barkhudarov, Sov. Phys. JETP 13, 1018 (1961).

${ }^{29}$ H. Heiselberg, C. J. Pethik, H. Smith, and L. Viverit, Phys. Rev. Lett. 85, 2418 (2000).

${ }^{30}$ R. Haussmann, W. Rantner, S. Cerrito, and W. Zwerger, Phys. Rev. A 75, 023610 (2007).

${ }^{31}$ D. S. Petrov, C. Salomon, and G. V. Shlyapnikov, Phys. Rev. Lett. 93, 090404 (2004); Phys. Rev. A 71, 012708 (2005).

${ }^{32}$ G. E. Astrakharchik, J. Boronat, J. Casulleras, and S. Giorgini, Phys. Rev. Lett. 93, 200404 (2004).

${ }^{33}$ G. E. Astrakharchik, J. Boronat, J. Casulleras, and S. Giorgini, Phys. Rev. Lett. 95, 230405 (2005).

${ }^{34}$ V. K. Akkineni, D. M. Ceperley, and N. Trivedi, arXiv:cond-mat/ 0608154 (unpublished). 
${ }^{35}$ Y. Nishida and D. T. Son, Phys. Rev. Lett. 97, 050403 (2006).

${ }^{36}$ Y. Nishida and D. T. Son, Phys. Rev. A 75, 063617 (2007).

${ }^{37}$ Y. Nishida, Phys. Rev. A 75, 063618 (2007).

${ }^{38}$ P. Nikolić and S. Sachdev, Phys. Rev. A 75, 033608 (2007).

${ }^{39}$ M. Y. Veillette, D. E. Sheehy, and L. Radzihovsky, Phys. Rev. A 75, 043614 (2007).

${ }^{40}$ A. Perali, P. Pieri, and G. C. Strinati, Phys. Rev. Lett. 93, 100404 (2004).

${ }^{41}$ P. Pieri, L. Pisani, and G. C. Strinati, Phys. Rev. B 72, 012506 (2005).

${ }^{42}$ A. L. Fetter and J. D. Walecka, Quantum Theory of ManyParticle Systems (McGraw-Hill, New York, 1971).

${ }^{43}$ M. Randeria, J.-M. Duan, and L.-Y. Shieh, Phys. Rev. B 41, 327 (1990).

${ }^{44}$ R. Combescot, Phys. Rev. Lett. 91, 120401 (2003).

${ }^{45}$ L. D. Landau and E. M. Lifshitz, Course of Theoretical Physics. Vol. 3: Quantum Mechanics: Non-relativistic Theory (Pergamon, Oxford, 1977).

${ }^{46}$ M. Holland, S. J. J. M. F. Kokkelmans, M. L. Chiofalo, and R. Walser, Phys. Rev. Lett. 87, 120406 (2001).

${ }^{47}$ J. N. Milstein, S. J. J. M. F. Kokkelmans, and M. J. Holland, Phys. Rev. A 66, 043604 (2002).

${ }^{48}$ G. M. Falco and H. T. C. Stoof, Phys. Rev. Lett. 92, 130401
(2004).

${ }^{49}$ S. J. J. M. F. Kokkelmans, J. N. Milstein, M. L. Chiofalo, R. Walser, and M. J. Holland, Phys. Rev. A 65, 053617 (2002).

${ }^{50}$ G. M. Falco and H. T. C. Stoof, Phys. Rev. A 75, 023612 (2007).

${ }^{51}$ G. M. Bruun, Phys. Rev. A 70, 053602 (2004).

${ }^{52}$ R. B. Diener and T. L. Ho, arXiv:cond-mat/0405174 (unpublished).

${ }^{53}$ S. Simonucci, P. Pieri, and G. C. Strinati, Europhys. Lett. 69, 713 (2005).

${ }^{54}$ J. von Stecher and C. H. Greene, Phys. Rev. A 75, 022716 (2007).

${ }^{55}$ T.-L. Ho, Phys. Rev. Lett. 92, 090402 (2004).

${ }^{56}$ G. B. Partridge, K. E. Strecker, R. I. Kamar, M. W. Jack, and R. G. Hulet, Phys. Rev. Lett. 95, 020404 (2005).

${ }^{57}$ M. H. Szymanska, K. Goral, T. Köhler, and K. Burnett, Phys. Rev. A 72, 013610 (2005).

${ }^{58}$ M. M. Parish, B. Mihaila, B. D. Simons, and P. B. Littlewood, Phys. Rev. Lett. 94, 240402 (2005).

${ }^{59}$ V. N. Popov, Functional Integrals in Quantum Field Theory and Statistical Physics (Reider, Dordrecht, 1983).

${ }^{60}$ R. Combescot, M. Yu. Kagan, and S. Stringari, Phys. Rev. A 74, 042717 (2006). 